\title{
Blunting of conical tips by surface diffusion
}

\author{
Catherine Lamstaes and Jens Eggers \\ Department of Mathematics, University of Bristol, University Walk, Bristol BS8 1TW, United Kingdom
}

(Received 3 February 2013; published 27 June 2013)

\begin{abstract}
We study the evolution of an initially conical metal surface when it is heated. For all cone angles $\alpha$ from close to zero to 90 degrees, self-similar solutions with rounded tips are found, whose radius of curvature scales like (time) $)^{1 / 4}$. For $\alpha \gtrsim 3^{\circ}$, theoretical profiles agree very well with experiment. For smaller cone angles, we find pronounced oscillations near the tip, which presumably are responsible for the experimentally observed fragility of such tips. The amplitude and wavelength of oscillations are characterized asymptotically.
\end{abstract}

DOI: 10.1103/PhysRevE.87.062408

PACS number(s): 68.03.Cd

\section{INTRODUCTION}

Pointed metal tips are used in the scanning tunneling microscope (STM), invented by G. Binning and W. Rohrer in $1982[1,2]$ to view surfaces at the atomic level. Similar tips are employed in the field ion microscope $[3,4]$ and the atom probe field ion microscope [5]. To operate, the STM requires the heating of the tip, close to the materiel's surface. A problem that arises during operation is that the tip blunts with time (see Fig. 1). One mechanism by which this blunting occurs is surface diffusion [6]. At sufficiently high temperatures (yet well below the melting temperature), atoms at the surface become mobile and diffuse so as to achieve a thermodynamically lower energy configuration. This process induces a surface transport of atoms away from regions of very high curvature (the tip apex) toward regions with low curvature, causing the tip to blunt.

The PDE describing surface diffusion was found by Mullins [8]. Nichols and Mullins [6] solved the equation numerically for a conical tip and found a sequence of shapes whose radius of curvature at the tip increases like $(B t)^{1 / 4}[9]$, where $B$ is a constant measuring the efficiency of surface transport, and $t$ is the time of blunting. Rescaling the results using this length scale leads to a collapsed profile (or similarity solution) that is characteristic of the opening angle. However, Nichols and Mullins found evidence that such similarity solutions only exist for angles $\alpha>\alpha_{c}$ above a critical angle $\alpha_{c} \approx 3^{\circ}$. Below $\alpha_{c}$, they observed solid "drops" being shed from the tip, similar to drops expected to form from a cylindrical rod, which is susceptible to the Rayleigh instability [10]. This process has been termed "spheroidization" [6]. Experimental results $[11,12]$ also point to a critical cone angle below which spheroidization occurs. Figure 2 shows experimental results for the blunting of conical tungsten tips for cone angles below (left) and above $3^{\circ}$ (right).

In this paper, we investigate the behavior of similarity solutions directly, by transforming the equation of motion to similarity variables and looking for stationary solutions of the resulting ODE. This permits us to investigate the limiting case of small cone angles much more accurately than before. Using a numerical procedure, we find continuous solutions for all angles, i.e., there is no evidence of a critical angle below which pinch-off occurs. For experiments in the regime of continuous blunting, we find excellent agreement between the experiments in Refs. [11,12] and our similarity solutions. However, for $\alpha \lesssim 3^{\circ}$, similarity solutions begin to form a bulbous head, separated from the cone by a thin neck. As $\alpha$ is decreased further, strong oscillations are observed behind the neck, which decay very slowly.

We then analyze this behavior analytically in the limit of small cone angles and find two different regions. Far away from the tip, oscillations are damped exponentially, and their wavelength increases like the distance from the tip to the power $1 / 3$. Toward the tip, on the other hand, the damping disappears and the wavelength depends only logarithmically on the distance from the tip. This behavior is confirmed by comparison to numerical solutions of the similarity equation.

The solutions found by us are relevant also to the breakup of an axisymmetric piece of material under surface diffusion [13]. It has been shown that the profile near the point of breakup is asymptotically conical, with an opening angle of $\alpha_{b}=46.04^{\circ}$ on both sides of the point of breakup. Then the postbreakup solution consists of two identical similarity solutions of blunting cones, with a corresponding cone angle $\alpha_{b}$ [14]. Combining both pre- and postbreakup solutions, we have thus constructed a unique continuation across the singularity.

In the next section, we recall the model equation for an axisymmetric body and derive the similarity equation. We explain our numerical method for solving the similarity equation and describe the results qualitatively for various values of the opening angle $\alpha$. In the third section, we describe the similarity shapes analytically, by calculating the behavior of small perturbations about a cone. Finally, we offer a brief discussion.

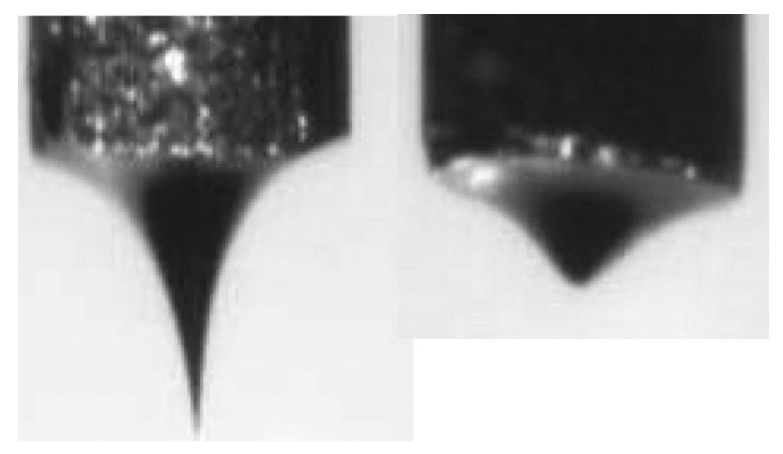

FIG. 1. Images of a STM tip (left) and a blunt STM tip (right) by Olfert [7]. 

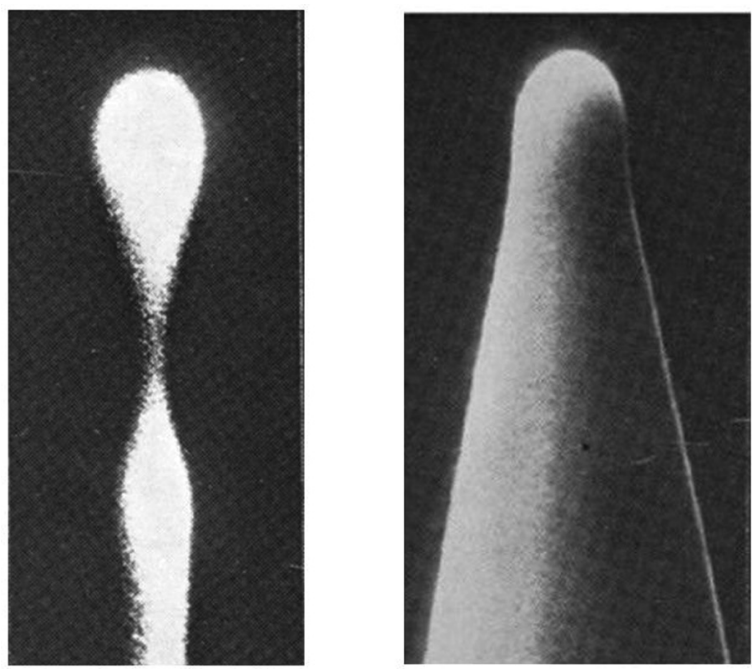

FIG. 2. Image of blunting of conical tungsten tips for (left) cone angle $\alpha=1^{\circ}$ and (right) cone angle $\alpha=11^{\circ}[12]$.

\section{MODEL AND SIMILARITY SOLUTION OF BLUNTING}

\section{A. Equation of motion}

The diffusion of atoms along a surface, driven by gradients of surface tension, is described by a surface current $\mathbf{J}[6,8]$ given by

$$
\mathbf{J}=-B \nabla_{s} \kappa,
$$

where $\kappa$ is (twice) the mean curvature, and $\nabla_{s}$ is the surface gradient. The constant $B$ has dimensions $\mathrm{cm}^{4} / \mathrm{s}$; a typical value of $B$ for a metal near the melting point is $B=10^{-18} \mathrm{~cm}^{4} / \mathrm{s}$ [8]. The shape evolution of a body of revolution can be described by a local radius function $h(z, t)$, where $z$ is the position along the axis of symmetry. Then, from Eq. (1), one finds [13,15] the equation of motion

$$
\frac{\partial h}{\partial t}=\frac{B}{h}\left(\frac{\partial}{\partial z}\left\{\frac{h}{\left[1+\left(\partial_{z} h\right)^{2}\right]^{1 / 2}} \frac{\partial \kappa}{\partial z}\right\}\right) .
$$

In terms of $h(z, t)$, the mean curvature is given by

$$
\kappa=\frac{1}{h\left[1+\left(\partial_{z} h\right)^{2}\right]^{1 / 2}}-\frac{\partial_{z z} h}{\left[1+\left(\partial_{z} h\right)^{2}\right]^{3 / 2}} .
$$

\section{B. Similarity description}

As an initial condition, we consider a conical tip with opening angle $\alpha$. Since a typical length scale after time $t$ is given by $(B t)^{1 / 4}$, we seek similarity solutions of the form

$$
h(z, t)=(B t)^{1 / 4} H(\xi), \quad \xi=\frac{z}{(B t)^{1 / 4}} .
$$

Substituting this into Eq. (2), we find the fourth order ODE

$$
\frac{1}{4} H-\frac{1}{4} \xi H^{\prime}=\frac{1}{H}\left\{\left[\frac{H}{Q^{1 / 2}}\left(\frac{1}{H Q^{1 / 2}}+\frac{H^{\prime \prime}}{Q^{3 / 2}}\right)^{\prime}\right]^{\prime}\right\}
$$

where $Q=1+H^{\prime 2}$ and ()$^{\prime}=[]^{\prime}=\frac{\partial}{\partial \xi}$. The similarity Eq. (5) is to be solved with the boundary condition $H(\xi)^{\prime}=\tan \alpha$ for $\xi \rightarrow \infty$. In practice, the tip of any realistic initial shape will be rounded on some scale $r$. This means that the self-similar dynamics described by Eq. (4) should apply after a time:

$$
t_{r}=r^{4} / B \text {. }
$$

For $t>t_{r}$ the typical scale of the solution has become larger than that of the initial rounding, so its effects should have been obliterated. We, therefore, expect our similarity solutions to represent faithfully full solutions of the PDE Eq. (2) for times $t \gtrsim t_{r}$.

\section{Numerical method}

We aim to solve Eq. (5) numerically, subject to the boundary condition of constant cone angle $\alpha$ at infinity, integrating from the (a priori unknown) tip position $\xi_{\text {tip }}$, where $H\left(\xi_{\text {tip }}\right)=0$. In view of Eq. (4), this means that the tip position $z_{\text {tip }}$ recedes according to

$$
z_{\text {tip }}=\xi_{\text {tip }}(B t)^{1 / 4}
$$

The larger $\xi_{\text {tip }}$, the faster the tip recedes; hence, we expect $\xi_{\text {tip }}$ to be a monotonically decreasing function of $\alpha$.

Since the slope of the profile is infinite at $\xi_{\text {tip }}$, it is convenient to transform to a new set of variables $V(\theta)$, as illustrated in Fig. 3:

$$
\begin{aligned}
& H(\xi)=V(\theta) \sin (\theta) \\
& \xi=V(\theta) \cos (\theta) .
\end{aligned}
$$

Now the tip apex is located at $\theta=0$, and using the symmetry of the new coordinates, we have $V(\theta)=V(-\theta)$. Thus, the initial conditions at $\theta=0$ are

$$
V(0)=\xi_{\text {tip }}, \quad V^{\prime \prime}(0)=\beta,
$$

while $V^{\prime}(0)=V^{\prime \prime \prime}(0)=0$. For each $\xi_{\text {tip }}, \beta$ is an unknown constant that needs to be determined.

To generate suitable initial conditions to integrate Eq. (5) numerically, we expand $V(\theta)$ into a power series in $\theta$, in which (for given $\xi_{\text {tip }}$ ) $\beta$ is the only parameter. Evaluating the expansion at a small angle $\theta_{i}$ (typically $10^{-4}$ ), and transforming back to Cartesian coordinates using Eq. (8), we are able to obtain initial conditions $H\left(\xi_{i}\right), H^{\prime}\left(\xi_{i}\right), H^{\prime \prime}\left(\xi_{i}\right), H^{\prime \prime \prime}\left(\xi_{i}\right)$ at a position $\xi_{i}$ close to $\xi_{\text {tip. }}$. We then integrated Eq. (5) to large values of $\xi$ using standard MAPLE routines. We checked that the numerical solution was independent of our choice of $\theta_{i}$.

We expect that for each value of $\xi_{\text {tip }}$, there will be a unique similarity solution, corresponding to a value of the opening angle $\alpha$. Indeed, in Sec. III we will show that for any profile

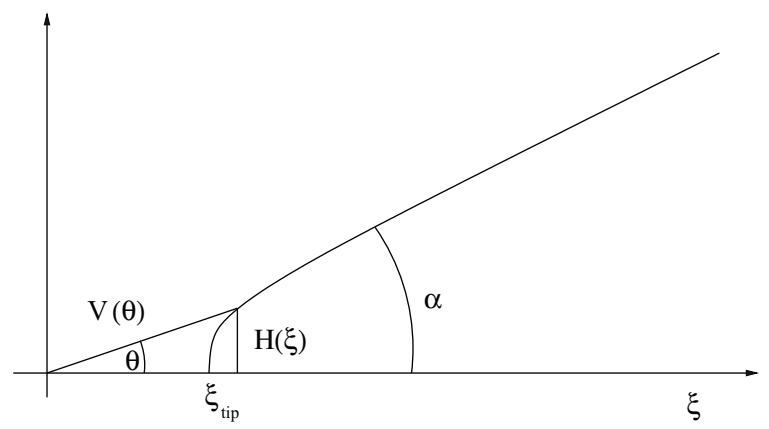

FIG. 3. Change of variables used near the tip. 
$H(\xi)$ approaching a cone, there is exactly one unstable mode that drives the solution from the linear asymptotics $H(\xi) \approx$ $\xi \tan \alpha$. This means that by adjusting $\beta$ suitably for each value of $\xi_{\text {tip }}$, we suppress the unstable direction and converge onto a linear profile $H(\xi)$. To find this value $\beta\left(\xi_{\text {tip }}\right)$, we proceed as follows:

We begin by fixing a value of $\xi_{\text {tip }}$, for which we want to find the solution. We find that for each value of $\beta$, either one of the following events can occur as we integrate to $\xi=\infty$ :

(1) The solution $H(\xi)$ blows up exponentially to infinity. This happens if $\beta>\beta\left(\xi_{\text {tip }}\right)$. We call this event +1 .

(2) $H(\xi)$ decays exponentially to zero. This occurs for $\beta<$ $\beta\left(\xi_{\text {tip }}\right)$. We call this event -1 .

We seek a solution between these two possible events, +1 or -1 , until $\beta$ converges to a constant value to 8 decimal pointsthis is the solution for $\beta$ that is used to solve the equation. The cone angle, $\alpha\left(\xi_{\text {tip }}\right)$, is determined from the taper's profile generated from the value $\beta\left(\xi_{\text {tip }}\right)$.

\section{Numerical results}

The numerical method described above solves Eq. (5) for any given $\xi_{\text {tip. }}$. The resulting profiles of blunted tips are shown in Fig. 4 for $\xi_{\text {tip }}=0.05$ to 12 . As expected, the smaller the cone angle, the faster the speed of retraction, i.e., the greater $\xi_{\text {tip }}$. It is seen that, for tips with cone angle $\alpha \gtrsim 8^{\circ}$, the radius increases monotonically as one proceeds from the apex [6]. In Fig. 5, we compare our numerical solutions to experimental profiles [12], which have evolved into a self-similar state, and find remarkable agreement between theory and experiment.

The postbreakup solution for an axisymmetric body consists of two conical tips, whose tip position recedes according to Eq. (7). From the stable prebreakup solution, one finds the cone angle to be $\alpha_{b}=46.04^{\circ}$, so it is appreciated from Fig. 4 that the shape is essentially a cone, with a slight rounding at the tip, on the scale $(B t)^{1 / 4}$. For $\alpha=\alpha_{b}$, we find $\xi_{\text {tip }}=1.13$, so after breakup the tip recedes like

$$
z_{\text {tip }}=1.13(B t)^{1 / 4} \text {. }
$$

For cone angles less than $8^{\circ}$, a neck forms near the apex. As the angle decreases further, the neck forms a complete oscillation. The number of oscillations increases as $\alpha \rightarrow 0$. This is to be expected, since a conical shape is susceptible to the Plateau-Rayleigh instability [10], which is a surface-

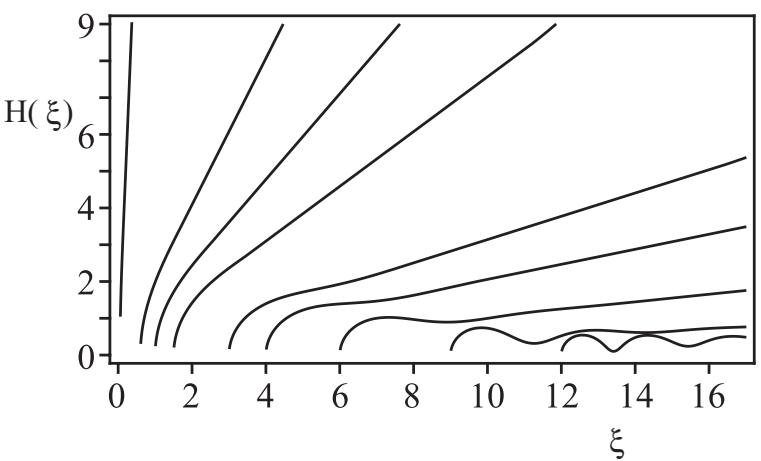

FIG. 4. Numerical solutions of $H(\xi)$ for $\xi_{\text {tip }}=0.05,0.6,1,1.5,3$, $4,6,9$, and 12 . The cone angles are $\alpha \cong 87^{\circ}, 63^{\circ}, 50^{\circ}, 37^{\circ}, 17^{\circ}$, $12^{\circ}, 5.9^{\circ}, 2.6^{\circ}$, and $1.4^{\circ}$, respectively.

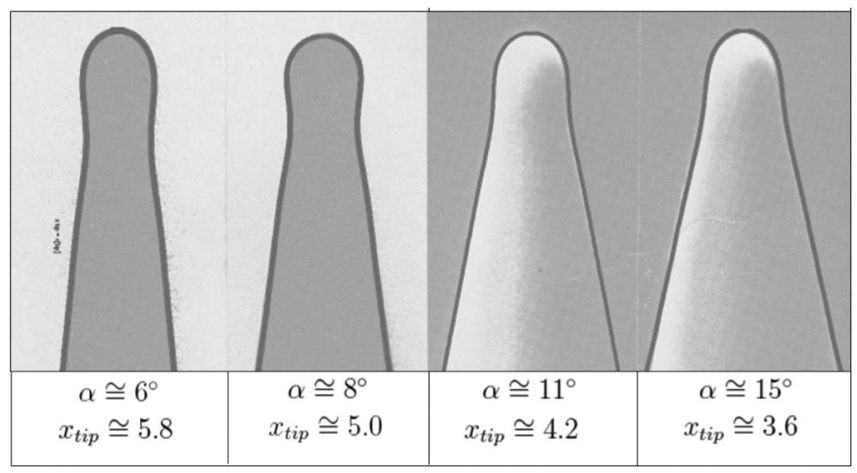

FIG. 5. Comparison of numerical simulations with experimental photographs [12] of blunting conical tungsten tips. Theory (dark gray lines) has been superimposed onto the photographs, whose contrast has been adjusted for clarity.

tension-driven instability leading to oscillations of the surface. For very small $\alpha$ (cf. Fig. 6), the surface profile consists of a taper with a very long sequence of slowly decaying oscillations with slowly increasing wavelength the further its distance from the apex. The oscillations never become disconnected and so breakup is not predicted by our similarity theory, in contrast to experimental observation that spheroidization occurs for $\alpha \lesssim 3^{\circ}$ [12]; see Fig 7. We will come back to this point in the Discussion.

In Fig. 8, we show the rescaled tip position $\xi_{\text {tip }}$ as a function of the slope $\tan \alpha$. In particular, we examine slopes very close to zero (a very sharp tip) and those approaching infinity (close to a flat wall). We find that as $\alpha \rightarrow 0$,

$$
\xi_{\text {tip }} \tan (\alpha)=\text { const } \approx 1.22
$$

while for $\alpha \rightarrow \pi / 2$,

$$
\xi_{\text {tip }}^{2} \tan (\alpha)=\text { const } \approx 3.48 \text {. }
$$

We do not have a full theoretical explanation for these scaling laws, but below we attempt to rationalize the observed behaviors.

\section{ASYMPTOTIC ANALYSIS}

We now develop an analytical description of the profile away from the tip. To this end we split up the profile into a

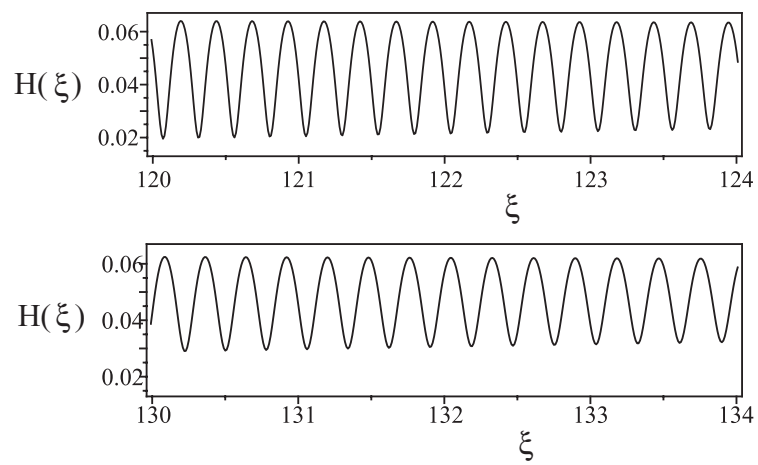

FIG. 6. Two sections of the profile $H(\xi)$ for $\xi_{\text {tip }}=100$, with cone angle $\alpha=0.019^{\circ}$. The amplitude is decreasing slowly, while the wavelength is increasing. 


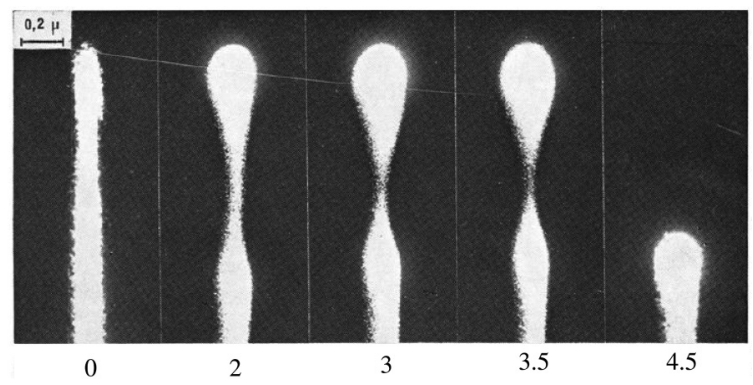

FIG. 7. Experimental images of the spheroidization of a conical tungsten tip of cone angle $\alpha=1^{\circ}$ [12]. Below each frame, the time in minutes from the beginning of the experiment.

base solution $\bar{H}(\xi)$ (which is varying linearly up to asymptotic corrections), and perturbations to it, which we describe by linear theory. Inspection of Eq. (5) shows that for $\xi \rightarrow \infty$, solutions can be expanded into the asymptotic series

$$
\bar{H}(\xi)=\sum_{i=0}^{\infty} \frac{a_{i}}{\xi^{4 i-1}} .
$$

The coefficients $a_{i}$, are found from substituting Eq. (12) into Eq. (5), and their values can be determined to arbitrarily high order. The first few are

$$
\begin{aligned}
& a_{0}=\tan (\alpha), \\
& a_{1}=\frac{1}{\tan (\alpha)\left[1+\tan (\alpha)^{2}\right]}, \\
& a_{2}=-\frac{21}{2} \frac{11 \tan (\alpha)^{2}+1}{\tan (\alpha)^{3}\left[1+\tan (\alpha)^{2}\right]^{3}} .
\end{aligned}
$$

In order to find the tip position $\xi_{\text {tip }}$, we formally have to solve $\bar{H}\left(\xi_{\text {tip }}\right)=0$, although, of course, the expansion Eq. (12)

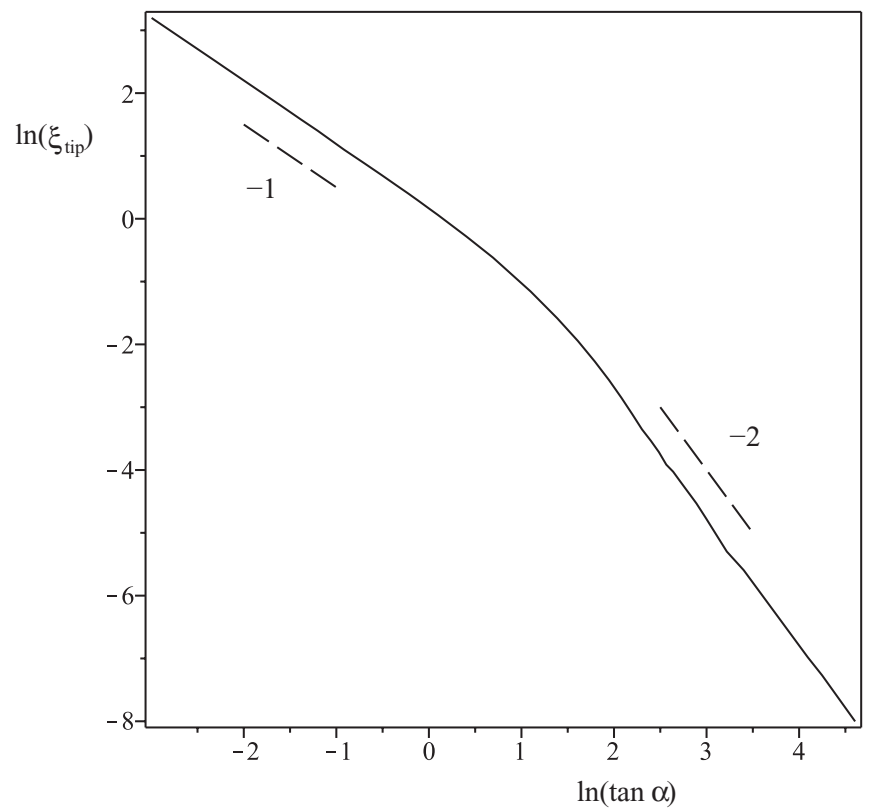

FIG. 8. Plot of $\ln \left(\xi_{\text {tip }}\right)$ against $\ln [\tan (\alpha)]$ from numerical solutions. breaks down near the tip. The scaling of $a_{i}$ for high orders is

$$
a_{i} \propto \begin{cases}(\tan \alpha)^{1-2 i} & \alpha \rightarrow 0 \\ (\tan \alpha)^{1-4 i} & \alpha \rightarrow \pi / 2 .\end{cases}
$$

But this means that if

$$
\xi_{\text {tip }} \propto \begin{cases}(\tan \alpha)^{-1 / 2} & \alpha \rightarrow 0 \\ (\tan \alpha)^{-1} & \alpha \rightarrow \pi / 2,\end{cases}
$$

all terms in the sum $\bar{H}\left(\xi_{\text {tip }}\right)$ scale in the same way as the limits $\alpha \rightarrow 0$ and $\alpha \rightarrow \pi / 2$ are approached. The scaling Eqs. (14) agree with the numerical observation Eqs. (10) and (11), but we are unable to determine the prefactors, since the expansion Eq. (12) is only asymptotic and does not converge near $\xi_{\text {tip }}$.

\section{A. Linear stability}

In order to understand the oscillations of the profile such as those shown in Fig. 6, we consider small perturbations about the base solution $\bar{H}(\xi)$ Eq. (12),

$$
H(\xi)=\bar{H}(\xi)+\delta(\xi),
$$

and linearize in $\delta(\xi)$. Before going through the detailed calculation, we summarize our results as presented in Fig. 9, which shows a comparison between a numerical solution for small $\alpha$ and asymptotic solutions of the linearized equation.

First, we analyze the far-field behavior for large $\xi$, for which oscillations are damped exponentially. Second, we show that for small angles $\alpha \ll 1$, another asymptotic behavior emerges, which applies to the tip region $\xi<\xi_{\text {cr }}$, where $\xi_{\text {cr }}$ is the crossover point between the tip and the far-field regions; below we will show that $\xi_{\text {cr }}=\left(4 / \alpha^{3}\right)^{1 / 4}$. In Fig. 9, the far field

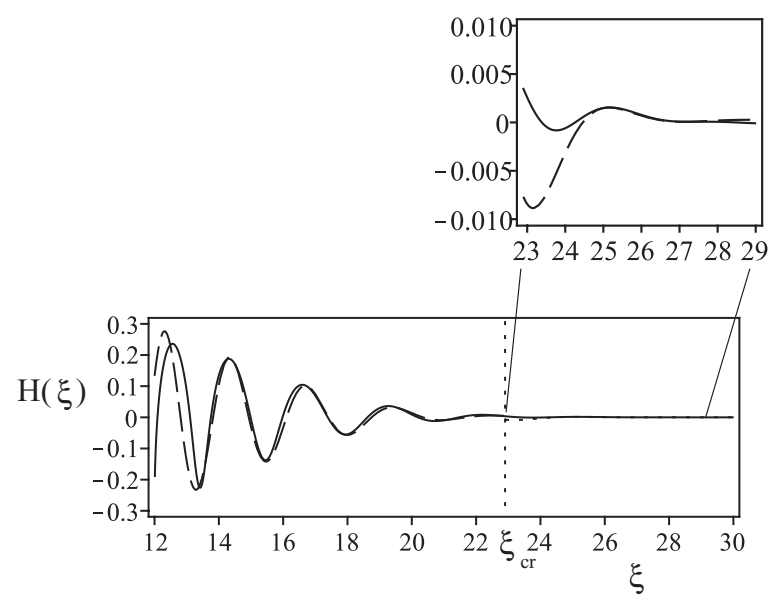

FIG. 9. Asymptotic and numerical solution for $\delta(\xi)$ for $\xi_{\text {tip }}=$ $12, \alpha=0.0243$. The full lines are numerical solutions of the similarity Eq. (5), with the base solution $\bar{H}(\xi)$ subtracted. The dashed lines are the results of our asymptotic calculations. We find two asymptotic regions, separated by a crossover value $\xi_{\text {cr }}=22.9$, marked by the dotted line. To the right of $\xi_{\text {cr }}$, we find a strongly damped far-field region, to the left of $\xi_{\mathrm{cr}}$, an oscillating region near the tip. To show some of the oscillations in the far-field region, we also include a magnified view. The free constants in Eqs. (35) and (36) that best fit the numerical solution have been found to be $A_{1}=0.21$, $\phi_{1}=\pi+0.7$ and $B_{1}=0.0025$, for $\xi<\xi_{\text {cr }}$ and $A_{2}=1.33 \times 10^{7}$, $\phi_{2}=0.6$ and $B_{2}=0.0025$ for $\xi>\xi_{\text {cr }}$. 
region is seen to the right of $\xi_{\text {cr }}=22.9$. Since the amplitude of oscillations is very small, we have expanded the scale in the inset. Toward the right of $\xi_{\mathrm{cr}}$, good agreement is found. On the other hand, in the tip region to the left of $\xi_{\mathrm{cr}}$, oscillations are only damped algebraically, and the wavelength is changing slowly, as seen on the left of Fig. 9. For the smallest cone angles, this leads to the extreme oscillations seen in Fig. 6.

We now set up the the general equations used to study the behavior of $\delta(\xi)$; to do so, we use a WKB ansatz. We then study the far-field behavior for large $\xi$ : this analysis applies to any angle. Next, we consider the case of small angles, for which another asymptotic region emerges, which we call the tip region.

Inserting Eq. (15) into the governing Eq. (5), and using

$$
\bar{H}(\xi)=\xi \tan \alpha+O\left(\xi^{-3}\right),
$$

we find to first order in $\delta$ :

$$
\begin{aligned}
& 4 \xi^{4} \cos (\alpha)^{4}\left[1-\cos (\alpha)^{2}\right] \delta^{\prime \prime \prime \prime}(\xi) \\
& \quad+\left\{8 \xi^{3} \cos (\alpha)^{4}\left[1-\cos (\alpha)^{2}\right]\right\} \delta^{\prime \prime \prime}(\xi) \\
& \quad+\left\{4 \xi^{2} \cos (\alpha)^{4}\left[1-2 \cos (\alpha)^{4}\right]\right\} \delta^{\prime \prime}(\xi) \\
& \quad+\left\{8 \xi \cos (\alpha)^{6}+\xi^{5}\left[1-\cos (\alpha)^{2}\right]\right\} \delta^{\prime}(\xi) \\
& \quad+\left\{\xi^{4} \cos \left[1-\cos (\alpha)^{2}\right]-8 \cos (\alpha)\right\} \delta(\xi)=0 .
\end{aligned}
$$

Using the WKB ansatz

$$
\delta(\xi)=e^{S(\xi)}
$$

Eq. (17) becomes

$$
\begin{aligned}
0= & \xi^{5} S^{\prime}\left[1-\cos (\alpha)^{2}\right] \\
& +4 \xi^{4} \cos (\alpha)^{6}\left(S^{\prime 4}+4 S^{\prime \prime \prime} S^{\prime}+6 S^{\prime \prime} S^{\prime 2}+S^{\prime \prime \prime \prime}+3 S^{\prime \prime 2}\right) \\
& -4 \xi^{4} \cos (\alpha)^{4}\left(S^{\prime 4}+4 S^{\prime \prime \prime} S^{\prime}+6 S^{\prime \prime} S^{\prime 2}+S^{\prime \prime \prime \prime}+3 S^{\prime \prime 2}\right) \\
& +\xi^{4}\left[\cos (\alpha)^{2}-1\right]+4 \xi^{3} \cos (\alpha)^{6}\left(2 S^{\prime 3}+6 S^{\prime \prime} S^{\prime}+2 S^{\prime \prime \prime}\right) \\
& +4 \xi^{3}\left[-\cos (\alpha)^{4}\left(2 S^{\prime 3}+6 S^{\prime \prime} S^{\prime}+2 S^{\prime \prime \prime}\right)+6 S^{\prime \prime} S^{\prime}\right] \\
& +4 \xi^{2}\left[\cos (\alpha)^{6}\left(-2 S^{2}\right)+\cos (\alpha)^{4}\left(S^{\prime \prime}+S^{\prime 2}\right)\right] \\
& -8 \xi^{2} \cos (\alpha)^{2} S^{\prime \prime}+8 \xi \cos (\alpha)^{6} S^{\prime}-8 \cos (\alpha) .
\end{aligned}
$$

To find the dominant balance, we assume that $S$ behaves like a power law:

$$
S(\xi)=a \xi^{b},
$$

where $a$ and $b$ are real constants.

\section{B. Far-field behavior}

One finds that for large $\xi$, the problem simplifies to finding the leading balance between the powers that characterize each term. These are

$$
\xi^{4+b}, 1, \xi, \xi^{b}, \xi^{2 b}, \xi^{3 b}, \xi^{4 b} .
$$

It follows that the only possible leading balance is

$$
\xi^{4+b} \sim \xi^{4 b} \gg 1,
$$

which is satisfied for $b=4 / 3$. This corresponds to the dominant balance

$$
-4 \xi^{4} S^{\prime 4}\left\{\cos (\alpha)^{4}\left[1-\cos (\alpha)^{2}\right]\right\} \sim \xi^{5} S^{\prime}\left[1-\cos (\alpha)^{2}\right]
$$

in Eq. (19), which implies

$$
\left[1-\cos (\alpha)^{2}\right] S^{\prime}(\xi)\left(\left\{\xi-4 \cos (\alpha)^{4}\left[S^{\prime}(\xi)\right]^{3}\right\}\right)=0 .
$$

This is a fourth-order equation in $S^{\prime}(\xi)$, as expected for a fourth-order problem. Solving for the third factor in Eq. (22) to vanish, we find three solutions for $S(\xi)$ of the form,

$$
S_{i}(\xi)=a_{i}\left\{\frac{3}{8}\left[\frac{2}{\cos (\alpha)^{4}}\right]^{\frac{1}{3}} \xi^{\frac{4}{3}}\right\}+R(\xi),
$$

where for $i=1,2,3$,

$$
a_{1}=-\frac{1}{2}+i \frac{\sqrt{3}}{2}, \quad a_{2}=-\frac{1}{2}-i \frac{\sqrt{3}}{2}, \quad a_{3}=1,
$$

and

$$
R(\xi) \ll \xi^{\frac{4}{3}} .
$$

Demanding the second factor in Eq. (22) to vanish, the last possible solution is $S(\xi)=b=$ const, and thus, $\delta(\xi)=$ const. Thus, Eq. (17) reduces to

$$
\left\{\xi^{4} \cos \left[1-\cos (\alpha)^{2}\right]-8 \cos (\alpha)\right\} \delta(\xi)=0,
$$

which is only satisfied for $\delta(\xi)=0$. In summary, we find that for large $\xi, \delta(\xi)$ can be expressed as a linear combination of the remaining eigenvalues,

$$
\delta(\xi)=A e^{S_{1}(\xi)}+B e^{S_{2}(\xi)}+C e^{S_{3}(\xi)},
$$

where $A, B, C$ are constants that need to be determined, and $S_{i}(\xi), i=1,2,3$ is as in Eq. (23). This corresponds to the eigenvalues found previously for the breakup problem [13].

We note that $S_{3}$ is the only eigenvalue with a positive real part, which leads to exponential departure from the conical solution. Thus, in agreement with our numerical observations, a single constant $\beta$ needs to be adjusted to ensure $C=0$, and to avoid the "blow up" events described in the numerical procedure. The remaining two oscillatory modes are heavily damped and thus converge quickly onto the linear base solution. Moreover, the prefactor [cf. Eq. (23)] depends on the cone angle $\alpha$ and goes to infinity as $\alpha \rightarrow \frac{\pi}{2}$. This makes the rate of decay even faster for $\alpha \rightarrow \frac{\pi}{2}$. Also, note that the wavelength of the oscillation increases like $\xi^{1 / 3}$. Figure 9 illustrates the match between the leading asymptotic solution with the numerical results for $\xi>\xi_{\text {cr }}=22.9$, where $\xi_{\text {cr }}$ is the crossover scale to be identified below.

After having found the leading order behavior for $\xi \rightarrow \infty$, we now reveal the general structure of the linear solution in this limit. For $C=0$, we find

$$
\delta(\xi)=A e^{S_{1}(\xi)}+B e^{S_{2}(\xi)},
$$

where

$$
S_{j}(\xi)=\sum_{n=0}^{\infty}\left(-b_{n} \xi^{\frac{4-4 n}{3}}+i \sqrt{3} c_{n}[j] \xi^{\frac{4-4 n}{3}}\right),
$$

for $j=1,2, c_{n}[1]=-c_{n}[2]$, and constants $A$ and $B$. Note that in calculating the coefficients, the full expansion Eq. (12) of the base solution has to be taken into account. The first three 
coefficients are

$$
\begin{aligned}
& b_{0}=c_{0}[1]=\frac{3}{16} \sqrt[3]{2}\left[\frac{1}{\cos (\alpha)}\right]^{\frac{4}{3}} \\
& b_{1}=-\frac{5}{3} \ln (\xi), \quad c_{1}[1]=0 \\
& b_{2}=c_{2}[1]=\frac{1}{72} 2^{\frac{2}{3}} \cos (\alpha)^{\frac{4}{3}} \frac{85 \cos ^{2}(\alpha)-94}{\sin ^{2}(\alpha)} .
\end{aligned}
$$

The coefficient $b_{0}$ corresponds to the solution already found in Eqs. (23) and (24).

\section{Tip region}

As illustrated in Fig. 6, numerical findings show that for small cone angles, there exist oscillations that are only weakly damped and whose wavelength changes only very slowly. Clearly, this behavior is not described by Eq. (23), and a new dominant balance must be sought, valid for small $\alpha$, and representing a distinguished scaling limit of $\alpha$ and $\xi$.

Returning to the linearized Eq. (17), for small $\alpha$ it simplifies to

$$
\begin{aligned}
0= & -4 \delta^{\prime \prime}(\xi)+8 \xi \delta^{\prime}(\xi)-8 \delta(\xi)+\alpha^{2}\left[-\xi^{4} \delta(\xi)+16 \delta(\xi)\right] \\
& +\alpha^{2}\left[16 \xi^{2} \delta^{\prime \prime}(\xi)-8 \xi^{3} \delta^{\prime \prime \prime}(\xi)-4 \xi^{4} \delta^{\prime \prime \prime \prime}(\xi)\right] \\
& +\alpha^{2}\left[\xi^{5} \delta^{\prime}(\xi)-24 \xi \delta^{\prime}(\xi)\right]+O\left(\alpha^{3}\right),
\end{aligned}
$$

and using Eq. (18), we get

$$
\begin{aligned}
0= & 4 \xi^{2}\left(S^{2}+S^{\prime \prime}\right)-8 \xi S^{\prime}+8+\alpha^{2}\left(-\xi^{5} S^{\prime}\right) \\
& +\alpha^{2} \xi^{4}\left(4 S^{\prime \prime \prime \prime}+16 S^{\prime \prime \prime} S^{\prime}+12 S^{\prime \prime 2}+24 S^{\prime \prime} S^{2}\right) \\
& +\alpha^{2}\left[\xi^{4}\left(4 S^{\prime 4}+1\right)+8 \xi^{3}\left(S^{\prime \prime \prime}+3 S^{\prime \prime} S^{\prime}+S^{\prime 3}\right)\right] \\
& +\alpha^{2}\left[-16 \xi^{2}\left(S^{\prime \prime}+S^{\prime 2}\right)+24 \xi S^{\prime}-16\right] .
\end{aligned}
$$

Since $\alpha \ll 1$, the possible dominant balances in Eq. (31) are between

$$
4 \xi^{2} S^{\prime 2}, \quad 4 \alpha^{2} \xi^{4} S^{\prime 4}, \quad-\alpha^{2} \xi^{5} S^{\prime},
$$

where the previous asymptotic balance Eq. (21) is between the last two terms, valid for all cone angles. However, for intermediate values of $\xi$, a different balance is possible, such that

$$
4 \xi^{2} S^{\prime 2} \sim 4 \alpha^{2} \xi^{4} S^{\prime 4} \gg-\alpha^{2} \xi^{5} S^{\prime} .
$$

This implies that

$$
S^{\prime} \approx \pm \frac{1}{\alpha \xi}
$$

and, thus,

$$
S(\xi, \alpha)= \pm \frac{1}{\alpha} \ln (\xi)+R(\xi, \alpha),
$$

where $R(\xi, \alpha) \ll \ln (\xi) / \alpha$. We will determine $R(\xi, \alpha)$, along with further asymptotic corrections, in the Appendix.

For the condition on $R(\xi, \alpha)$ to be valid, we need $4 \alpha^{2} \xi^{4} S^{\prime 4} \gg-\alpha^{2} \xi^{5} S^{\prime}$, which implies

$$
\xi_{\mathrm{cr}}=\left(\frac{4}{\alpha^{3}}\right)^{\frac{1}{4}} \gg \xi,
$$

which fixes the crossover scale between the far-field and tip regions. Table I reports typical values of $\xi_{\mathrm{cr}}$, demonstrating
TABLE I. Crossover values $\xi_{\text {cr }}$ for different angles

\begin{tabular}{lcc}
\hline \hline$\xi_{\text {tip }}$ & Cone angle $\alpha(\mathrm{Rad})$ & $\xi_{\text {cr }}$ \\
\hline 12 & 0.0243 & 22.9 \\
70 & 0.000697 & 330 \\
100 & 0.000335 & 571 \\
\hline \hline
\end{tabular}

that for small $\alpha$ there is wide range of $\xi$ values over which the alternative balance is possible.

\section{Comparison to numerical results}

In summary, as the cone angle decreases to zero, the profile of the taper can be divided into two regions: the first consisting of slowly decaying oscillations, whose wavelength changes logarithmically, and the second consisting of strongly damped oscillations. In order to compare to numerics, we have to adjust the amplitudes of the two modes, or alternatively, one amplitude and a phase. We also allow for a small shift in the baseline, thus arriving at

$$
A_{1} e^{-\frac{\xi^{4} \alpha^{2}}{32}} \cos \left[\frac{1}{\alpha} \ln (\xi)+\phi_{1}\right]+B_{1}
$$

for $\xi_{\text {tip }}<\xi<\xi_{\text {cr }}$, and

$$
A_{2} \xi^{-\frac{5}{3}} e^{-G \xi^{\frac{4}{3}}} \cos \left(G \sqrt{3} \xi^{4 / 3}+\phi_{2}\right)+B_{2}
$$

for $\xi>\xi_{\text {cr }}$, where we have defined $G=3 \sqrt[3]{2} / 16$. As shown in Fig 9, we find good agreement both in the tip region and in the far-field region.

\section{DISCUSSION}

The main motivation for the present work was the original claim [6] of a transition between blunting (the tip remaining continuous) and spheroidization (the tip shedding drops in a discrete, but periodic fashion) near $\alpha=3^{\circ}$. Such a transition might be expected, since for small angles the cone becomes close in shape to a cylinder, which is susceptible to the PlateauRayleigh instability [10]. Namely, there will be an outflux of material from the neck regions of high mean curvature to the thick node regions. However, this effect is counterbalanced by a convective flux from the bulbous end, which retreats at the speed of the tip:

$$
\dot{z}_{\text {tip }}=\frac{\xi_{\text {tip }}}{4}\left(\frac{B}{t^{3}}\right)^{1 / 4} .
$$

But, $\xi_{\text {tip }}$ increases with decreasing $\alpha$, thus counterbalancing the Plateau-Rayleigh instability.

The similarity solutions found in our study do not exhibit any transition at small angles, but continuous solutions exist for all angles. This shows that retraction of the tip always overpowers the Plateau-Rayleigh instability. However, selfsimilar shapes show a pronounced bulbous head appearing near the "transition" value of $\alpha$. A closer inspection of the original paper [6] shows that pinch-off was documented only for $\alpha=0$ (a cylinder), while a simulation for $\alpha=2^{\circ}$ shows the formation of a pronounced minimum behind the head. This observation is consistent with our results for a self-similar blunting dynamics, without actual pinch-off occurring. 
In practice, however, the pronounced oscillations observed for smaller angles may well lead to pinch-off, as found in Ref. [12], since similarity solutions can only expected to be a faithful description of the full dynamics in the long time limit $t \gtrsim t_{r}$; cf. Eq. (6). For $t \lesssim t_{r}$, the dynamics may still depend on the details of the initial conditions. Thus, a few drops might be shed as a transient effect, until a self-similar evolution develops. Inhomogeneities in the sample, as well as competing transport mechanisms (e.g., evaporation-condensation [8]) might also be responsible.

In conclusion, we have formulated a similarity theory for the blunting of a cone, finding excellent agreement with experiment, except at very small angles. An analysis of the limit of small angles reveals a novel behavior near the tip, exhibiting oscillations that are only weakly damped. This regime might be a partial explanation for the "spheroidization" observed in experiment.

\section{APPENDIX: CORRECTIONS TO TIP OSCILLATIONS}

Inserting Eq. (33) into Eq. (31), the next-to-leading order balance is

$$
\frac{i 8 \xi}{\alpha} R^{\prime} \sim \mp i \xi^{4} \alpha \gg-\xi^{5} \alpha^{2} R^{\prime} .
$$

This implies that

$$
R(\xi, \alpha)=-\frac{\xi^{4} \alpha^{2}}{32}+D(\xi, \alpha)
$$

where $D(\xi, \alpha) \ll \frac{\xi^{4} \alpha^{2}}{32}$, which is valid as long as

$$
\xi \ll\left(\frac{8}{\alpha^{3}}\right)^{\frac{1}{4}} \text {. }
$$

This is consistent since the right-hand side of Eq. (A3) is greater than $\xi_{\mathrm{cr}}$.

When evaluating $D(\xi, \alpha)$, we find that two different balances in the region $\xi<\xi_{\text {cr }}$ are possible, namely

$$
\frac{i 8 \xi}{\alpha} D^{\prime} \sim-\frac{3}{16} \xi^{8} \alpha^{4},
$$

if $\xi \gg \widetilde{\xi}_{\mathrm{cr}}$, or

$$
\frac{i 8 \xi}{\alpha} D^{\prime} \sim \frac{13}{2} \xi^{4} \alpha^{2},
$$

if $\xi \ll \widetilde{\xi}_{\text {cr. }}$. The marginal balance point between both balances occurs at

$$
\widetilde{\xi}_{\text {cr }}=\left(\frac{104}{3}\right)^{\frac{1}{4}} \alpha^{-1 / 2} .
$$

But, since according to Eq. (11) $\xi_{\text {tip }} \propto \alpha^{-1 / 2}$, Eq. (A4) is always valid, except in a region approaching the apex. Solving for $D(\xi, \alpha)$, from Eq. (A4) we get

$$
D(\xi, \alpha)=\mp i \frac{3}{256} \xi^{8} \alpha^{5} .
$$

When a similar analysis is pursued to higher order, more partitions of regions of different dominant balances occur as the size of $\xi$ increases.
[1] G. Binnig, H. Rohrer, C. Gerber, and E. Weibel, Appl. Phys. Lett. 40, 178 (1982)

[2] G. Binnig, H. Rohrer, C. Gerber, and E. Weibel, Phys. Rev. Lett. 49, 57 (1982).

[3] E. W. Müller, Science 149, 591 (1965).

[4] M. Drechsler, Surf. Sci. 70, 1 (1978).

[5] E. W. Müller, J. A. Panitz, and S. B. McLane, Rev. Sci. Instrum. 39, 83 (1968).

[6] F. A. Nichols and W. W. Mullins, J. Appl. Phys. 36, 1826 (1965).

[7] N. Olfert, Presentation at Surface Canada Conference, Vancouver, 2004 (unpublished).
[8] W. W. Mullins, J. Appl. Phys. 28, 333 (1957).

[9] C. Herring, J. Appl. Phys. 21, 301 (1950).

[10] J. Eggers and E. Villermaux, Rep. Progr. Phys. 71, 036601 (2008).

[11] M. Drechsler, A. Piquet, R. Uzan, and V. Thien Binh, Surf. Sci. 14, 457 (1969).

[12] M. Drechsler, A. Piquet, H. Rouz, R. Uzan, and V. Thien Binh, Surf. Sci. 25, 348 (1971).

[13] A. J. Bernoff, A. L. Bertozzi, and T. P. Witelski, J. Stat. Phys. 93, 725 (1998).

[14] J. Eggers, Phys. Fluids 7, 941 (1995).

[15] J. Eggers, Phys. Rev. Lett. 80, 2634 (1998). 